\title{
Single Vehicular Delivery of siRNA and Small Molecules to Control Stem Cell Differentiation
}

\author{
Shreyas Shah, ${ }^{\ddagger}$ Aniruddh Solanki, ${ }^{\ddagger}$ Pijus K. Sasmal, and Ki-Bum Lee*
}

Department of Chemistry \& Chemical Biology , Rutgers, The State University of New Jersey, Piscataway, New Jersey 08854, United States

Supporting Information

ABSTRACT: Achieving a controlled and reproducible means to direct stem cell differentiation is the single most critical concern scientists have been trying to address since the discovery of stem cells. In this regard, the use of small molecules and RNA interference offers unique advantages by targeting different cellular mechanisms. Our cyclodextrin-modified dendritic polyamine construct (termed DexAM) combines the unique properties of two distinct chemical moieties in a single delivery vehicle. DexAM is a single vehicle that not only solubilizes hydrophobic small molecules in physiological solutions but also forms complexes with siRNA molecules, making it an attractive delivery system for controlling stem cell differentiation. Herein, we report the synthesis and application of DexAM to simultaneously deliver hydrophobic small molecules and siRNA into neural stem cells to significantly enhance their neuronal differentiation.

tem cells are becoming increasingly attractive as treatment $\checkmark$ options for regenerative medicine due to their ability to differentiate into specialized cells and tissues of interest. However, achieving a controlled and reproducible means to direct stem cell differentiation is the single most critical concern scientists have been trying to address since the discovery of stem cells. In this regard, a chemical approach has been widely applied wherein small molecules are used to modulate specific signaling cascades and eventually gene expression within the cell. For instance, novel small molecules which can control a variety of stem cell fates and functions including stem cell pluripotency, differentiation, and reprogramming have been screened and identified. ${ }^{1-3}$ Examples of such small molecules that have been used to modulate stem cell phenotypes include retinoic acid, cytidine analogues, histone-deacetylase inhibitors, and protein kinase inhibitors. ${ }^{2}$ The use of small molecules to regulate stem cell behavior is particularly advantageous as they provide a high degree of temporal control over protein function by either rapid inhibition or activation of single or multiple targets within a protein family. ${ }^{3}$ In addition to the chemical approach, a more delicate control of gene expression has been demonstrated using RNA interference (RNAi). RNAi has been widely used for treating genetic diseases and cancers. ${ }^{4,5}$ Many studies in the past decade have even demonstrated this strategy to be equally important for directing stem cell differentiation. ${ }^{6}$

However, a majority of small molecules tend to be very hydrophobic and lack solubility in physiological solutions, which can greatly impair its delivery and efficacy. ${ }^{7}$ As a result, organic solvents such as dimethyl sulfoxide (DMSO) are often used to dissolve such compounds. These solvents have shown to be cytotoxic and require careful dilution to avoid stem cell death and undesired side effects. ${ }^{8}$ Similarly, a major challenge for delivering siRNA into stem cells is designing a robust and reliable delivery system, ${ }^{9}$ such that it allows high cellular viability over an extended time period after transfections to ensure the differentiated cellular subtypes can be effectively used for further studies (e.g., in vivo transplantation, animal studies, etc). ${ }^{10}$ Therefore, we believe that designing a delivery system which could solubilize hydrophobic small molecules in physiological solutions and at the same time form complexes with siRNA molecules would be significantly advantageous. This delivery system would enable the simultaneous delivery of siRNA and hydrophobic small molecules into stem cells to enhance stem cell differentiation with minimal cytotoxicity.

Toward this goal, herein, we demonstrate the synthesis and application of a multifunctional vehicle for the simultaneous delivery of siRNA molecules and hydrophobic small molecules to direct the differentiation of a multipotent adult stem cell line (Figure 1).

Our delivery system is a single delivery platform which provides: (i) the ability to simultaneously deliver nucleic acids and hydrophobic small molecules to achieve a synergistic

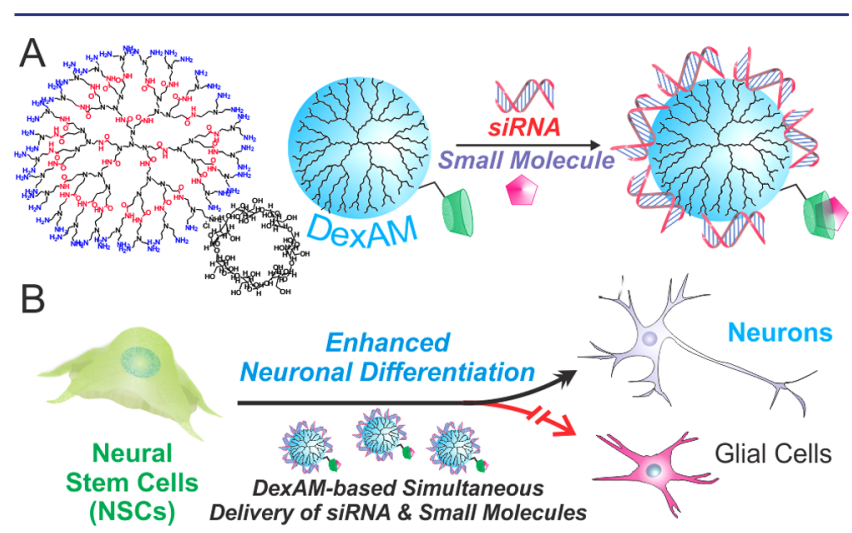

Figure 1. (A) DexAM is complexed with siRNA via electrostatic interaction and the small molecule via $\beta$-cyclodextrin encapsulation. (B) DexAM constructs are delivered to NSCs to enhance differentiation into neurons.

Received: July 18, 2013

Published: October 9, 2013 
enhancement in stem cell differentiation, (ii) high transfection efficiency of siRNA, and (iii) minimal cytotoxicity, allowing stem cells to differentiate over longer periods. While such dual delivery platforms are widely prevalent for inducing apoptosis of cancer cells, ${ }^{11,12}$ as far as we know, this is the first demonstration showing the application for inducing stem cell differentiation. Unlike other dual delivery systems, we believe our platform is significantly novel because it not only allows for the simultaneous delivery of factors to direct stem cell differentiation, but also ensures long-term cell growth and survival.

Our cyclodextrin-modified dendritic polyamine construct (termed DexAM) combines the unique properties of two distinct chemical moieties in a single delivery vehicle. First, the $\beta$-CD component serves to improve the solubility of hydrophobic compounds. ${ }^{13}$ Cyclodextrins are cyclic oligosaccharides containing a hydrophilic exterior and hydrophobic interior, wherein the inclusion of hydrophobic moieties within the inner cavity serves to enhance the solubility, stability and bioavailability of hydrophobic small molecules. ${ }^{14}$ Given that most small molecules and drugs have poor water solubility and necessitate the use of cytotoxic organic solvents, such as $\mathrm{DMSO}$, the presence of $\beta$-CD in our delivery vehicle enhances water solubility of drugs/small molecules and allows for optimal cellular uptake and drug efficacy while avoiding the use of cytotoxic solvents. Second, the dendritic polyamine backbone provides a high density of positive surface charge, which can be used to condense negatively charged nucleic acids into cationic complexes. This would allow for effective intracellular delivery and endosomal escape within the stem cell. ${ }^{15}$ While maintaining a high degree of positive charge is crucial for proper complexation with siRNA molecules, the primary amines have been reported to interact with cellular components and impart undesired side-effects and cytotoxicity. ${ }^{16}$ Therefore, it is critical to achieve a balance between the cytotoxicity and complexation capacity of our DexAM construct.

The DexAM molecule was synthesized by using a multistep solution- and solid-phase synthesis (see Supporting Information (SI) for detailed experimental methods) developed by our group in a previous study. ${ }^{17}$ Briefly, the dendritic polyamine backbone was synthesized by the Michael addition of tris (2aminoethyl)amine and methyl acrylate, followed by amidation of the amino esters generated after Michael addition (Figure 2).

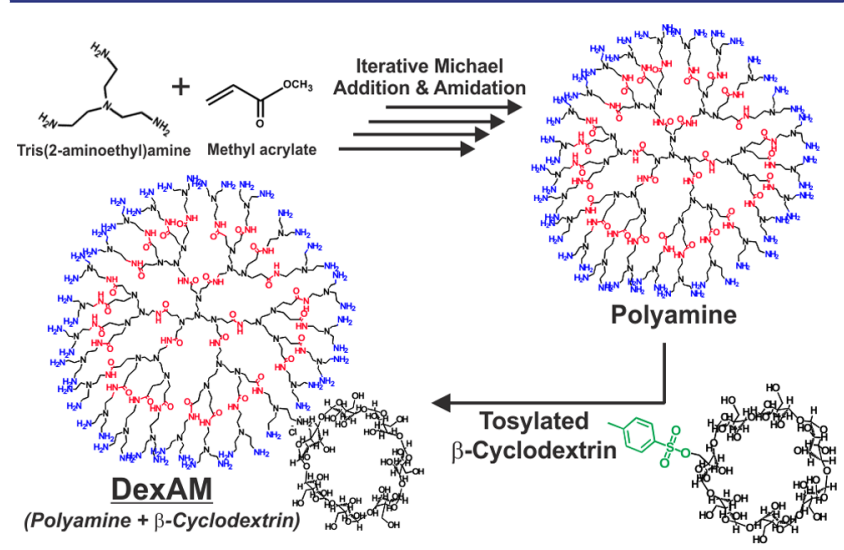

Figure 2. Polyamine backbone is initially synthesized by the iterative Michael addition of tris(2-aminoethyl)amine and methyl acrylate, followed by amidation. Tosylated $\beta$-cyclodextrin is then attached to form the final DexAM molecule.
Thereafter, this synthetic route (Michael addition followed by amidation) was repeated to yield a polyamine backbone consisting of 48 primary amines. The conjugation of $\beta$-CD to the polyamine backbone was performed by the tosylation of $\beta$ $\mathrm{CD}$, followed by nucleophilic addition to the amine group. By integrating the peaks of the $\beta$-CD protons (49 methylene protons) and the polyamine protons (161 methylene protons), we found that the $\beta$-CD was covalently bound to the polyamine in a molar ratio of $3: 1$. This molar ratio was found to be optimal, providing a good balance between the high loading capacity of the hydrophobic small molecule and the zeta potential required for siRNA complexation and delivery, as described below. This yielded our final DexAM molecule, which was used for the dual delivery of siRNA and small molecules to neural stem cells (NSCs).

We optimized the siRNA delivery using DexAM by suppressing the expression of green fluorescent protein (GFP) in NSCs that were genetically modified to express GFP (Figure 3a). However, prior to knocking down GFP
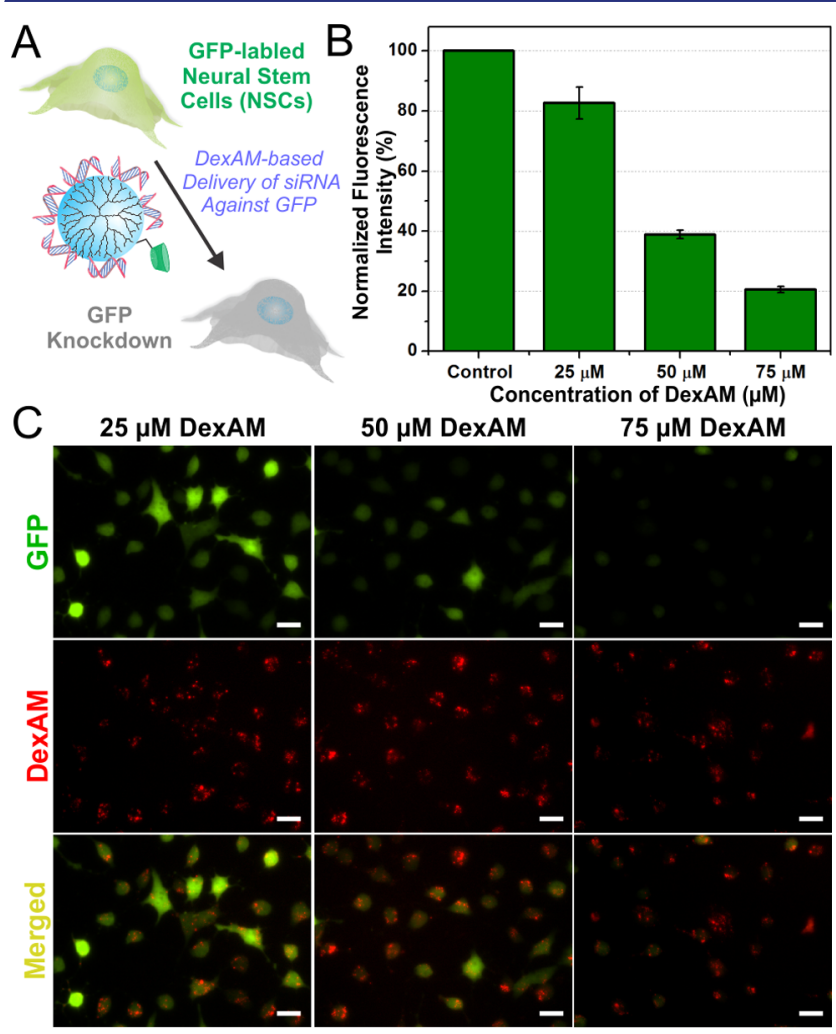

Figure 3. (A) Scheme depicting the delivery of DexAM complexed with siRNA targeting GFP to NSCs. (B) Quantitative comparison of the percentage of GFP knockdown at $96 \mathrm{~h}$ in NSCs with varying concentrations of DexAM. (C) Fluorescence images of cell with varying concentrations of DexAM showing GFP expression (green) and dye-labeled DexAM (red) at $96 \mathrm{~h}$. Scale bars: $20 \mu \mathrm{m}$.

expression within the NSCs by delivering siRNA using DexAM, we evaluated the capability of DexAM to spontaneously form complexes with the negatively charged siRNA. Using a wellestablished dye exclusion assay (picogreen assay), we identified a range of DexAM concentrations which showed about $75 \%$ of the siRNA binding to the DexAM molecule (Figure S1).

Thereafter, varying concentrations of the DexAM molecule were mixed with a given amount of GFP siRNA (100 pmol) to form the DexAM-siRNA complexes. The zeta potential of the 
resulting complexes was found to be in the range of $10-12 \mathrm{mV}$ at $\mathrm{pH} 7.4$, while exhibiting hydrodynamic diameters of 300$350 \mathrm{~nm}$. In order to visualize the efficiency of delivery, the DexAM molecule was conjugated with a fluorescent dye (AlexaFluor 594) prior to complexation with the GFP siRNA. The decrease in green fluorescence intensity was monitored using florescence microscopy over a time period of 48-96 h (Figure 3b,c). Successful transfection of the DexAM-siRNA constructs was evident as visualized by the presence of the dye fluorescence expression (conjugated to DexAM) in the NSCs (Figure 3c). The siRNA transfection efficiency and distribution within the cellular cytoplasm were further confirmed by delivering dye-labeled siRNA (Silencer negative control Cy3labeled siRNA, Ambion) complexed with unlabeled-DexAM (Figure S2). We observed a dose-dependent knockdown of GFP expression, wherein the NSCs treated with $75 \mu \mathrm{M}$ DexAM (complexed with GFP siRNA) showed a significant $80 \%$ knockdown in GFP intensity after $96 \mathrm{~h}$ of siRNA treatment compared to the untreated control cells (Figure $3 \mathrm{~b}$ ). In contrast, maximal GFP knockdown of only $33 \%$ was observed with commercial transfection reagents at the recommended doses (Figure S3). The high efficiency of siRNA delivery and knockdown by DexAM can be attributed to the presence of the highly dense primary amine groups on the surface in addition to the highly symmetrical and three-dimensional spherical structure of DexAM. ${ }^{18}$

While we achieved a remarkable knockdown efficiency with the DexAM-siRNA constructs, maintaining high cellular viability was equally important. Evaluating the cytotoxicity of the DexAM molecules is especially critical for applications in stem cell biology, which generally requires both longer time periods of culture (at least one week) and differentiated subtype specific cells that are functionally active for subsequent in vitro and in vivo studies. We assessed the cytotoxicity of the DexAM molecules using the MTS assay, which confirmed that our delivery vehicle shows negligible toxicity (Figure S4). In addition, NSCs treated with DexAM for siRNA delivery showed much higher cellular viability compared to those treated with commercially available transfection reagents at the recommended doses (Figure S5). While cationic polyaminebased polymers have been observed to show dose-dependent cytotoxicity in mammalian cells, we believe the biocompatibility of our DexAM constructs is due to the presence of $\mathrm{CD}$, which can reduce the nonspecific binding of the DexAM with cellular proteins and components. ${ }^{16,19}$

Having demonstrated the efficient gene silencing capability of DexAM by delivering siRNA against GFP, we focused on using our delivery vehicle for codelivering siRNA and small molecules to enhance the neuronal differentiation of NSCs. For the siRNA molecule, we designed a sequence to knockdown a well-established transcription factor known as SOX9, which acts as a switch between neuronal and glial differentiation. ${ }^{20}$ When the SOX9 gene is "turned on," a higher percentage of NSCs differentiate into astrocytes (glial cells), and when "turned off," a higher percentage of NSCs differentiate into neurons. ${ }^{21}$ For the small molecule, we selected the well-known vitamin A derivative all-trans retinoic acid (RA), which is known to play a crucial role in central nervous system development. RA functions by binding to RA receptors (RARs) in the cellular nucleus to form a ligand-receptor complex, which in turn binds to RA response elements to activate gene expression. ${ }^{22}$ However, this activation first requires the efficient intracellular delivery of RA into the cytoplasm, whereby the binding of RA to cytoplasmic proteins facilitates the above-mentioned downstream effects in the nucleus. Previous studies have reported that the treatment of RA tends to upregulate the expression of a variety of neuronal genes in cultured stem cells. ${ }^{21,23}$ Interestingly, exogenous expression of a SOX9 gene prior to the RA treatment was found to actually counteract the neuronal-promoting effect caused by RA, by suppressing neuronal gene expression. ${ }^{21}$ SOX9 is thus known to actively induce glial traits while simultaneously repressing neuronal traits. The knockdown of SOX9 using siRNA (siSOX9) and exposure to RA in NSCs has each individually been demonstrated to induce NSC differentiation into neurons. However, the two factors have varying mechanisms of action and target different signaling pathways in NSCs: RA forms a complex to modulate gene expression at the transcriptional level in the nucleus, while siSOX9 forms a RNA-induced silencing complex to modulate gene expression at the posttranscriptional level in the cytoplasm. Thus, we hypothesized that the simultaneous treatment with RA and suppression of SOX9 could greatly enhance the neuronal differentiation of NSCs. To this end, we utilized our DexAM construct to facilitate the codelivery of RA and siSOX9 (Figure 4a).

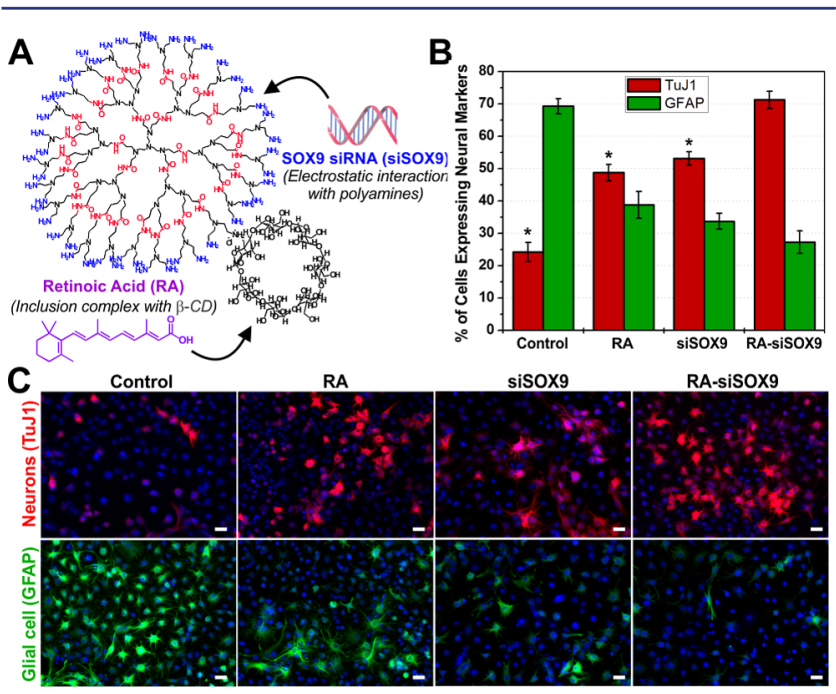

Figure 4. (A) Complexation to DexAM. (B) Quantification of the percentage of cells expressing neuronal TuJ1 and glial GFAP at day 6 . Student's unpaired $t$ test was used for evaluating the statistical significance for cells stained for TuJ1, compared to the RA-siSOX9 treatment $(*=P<0.001)$. (C) Fluorescence images of cells stained for the nucleus (blue), TuJ1 (red, top row), and GFAP (green, bottom row) at day 6. Scale bars: $20 \mu \mathrm{m}$.

RA has been used in previous studies for inducing neuronal differentiation, but given that it is extremely hydrophobic, it requires the use of toxic organic solvents like DMSO for delivery. For our studies, RA was loaded within the $\beta$-CD cavity of DexAM under reflux conditions to obtain highly watersoluble complexes, thus evading the use toxic organic solvents (see SI for details). By refluxing varying equivalents of RA with a given constant amount of DexAM, the RA encapsulation efficiency per $\beta$-CD was determined to be $\sim 77 \%$ (Figure S6).

Thereafter, the RA-loaded DexAM (2 $\mu \mathrm{M}$ RA) was complexed with the negatively charged siRNA (100 pmol; as described earlier) to form the DexAM constructs containing the siSOX9 and RA in a single delivery vehicle (Figure 4a). The NSCs were plated into culture plates at day 0 , followed by 
transfection with the DexAM-RA-siSOX9 constructs at day 1 . To confirm our hypothesis that the simultaneous delivery of RA and siSOX9 would enhance neuronal differentiation, we also transfected NSCs with (i) DexAM complexed with RA (and Silencer negative control siRNA to maintain the charge ratio), (ii) DexAM complexed with only siSOX9 (without RA). At day 6 , we quantified the extent of differentiation by immunostaining for neuronal (TuJ1) and glial (GFAP) markers (Figure 4b,c). The control (untreated) cells showed the majority of the NSCs differentiated into glial cells $(\sim 70 \%)$ vs neuronal cells $(\sim 25 \%)$, which is an expected differentiation pattern as observed previously. ${ }^{24}$ Conditions containing DexAM delivery of only RA and only siSOX9 resulted in about a 2 -fold increase in neuronal differentiation compared to the control, showing $\sim 49 \%$ and $53 \%$ of the cells expressing the neuronal marker $\mathrm{TuJ} 1$, respectively (Figure $4 \mathrm{~b}$ ). Compared to the various control treatment conditions (Figure S7), the simultaneous delivery of RA and siSOX9 to the NSCs using our DexAM molecule resulted in a remarkable increase in neuronal differentiation, wherein in $\sim 71 \%$ of the cells were expressing TuJ1 (Figure 4b). The enhanced neuronal differentiation using the combined DexAM-RA-siSOX9 construct was found to be statistically significant, confirming the synergistic effect of combining RA and siSOX9 for a single treatment to control NSC behavior.

In summary, the introduction of exogenous nucleic acids combined with small molecules into stem cells represents a powerful approach for manipulating stem cell behaviors. This is the first demonstration where a delivery platform capable of efficient and simultaneous delivery of siRNAs and hydrophobic small molecules has been shown to direct stem cell differentiation. Our synthetic methodology allows for facile manipulation of the core chemical structure to attain remarkable transfection efficiency while maintaining negligible cytotoxicity, thus allowing long-term cell survival. Moreover, our multimodal delivery vehicle enables a methodology by which we can target multiple signaling pathways and mechanisms to successfully enhance the neuronal differentiation of neural stem cells. With the increasing interest in achieving precise control over stem cell differentiation, our DexAM delivery system serves as a step toward bridging the gap between basic science and clinically relevant treatments.

\section{ASSOCIATED CONTENT}

\section{S Supporting Information}

Experimental details and characterization data. This material is available free of charge via the Internet at http://pubs.acs.org.

\section{AUTHOR INFORMATION}

\section{Corresponding Author}

kblee@rutgers.edu

\section{Author Contributions}

These authors contributed equally.

\section{Notes}

The authors declare no competing financial interest.

\section{ACKNOWLEDGMENTS}

K.-B.L. acknowledges financial support from the $\mathrm{NIH}$ Director's Innovator Award (1DP20D006462-01), the N.J. Commission on Spinal Cord grant (CSR13ERG005), and the Rutgers Faculty Research Grant Program. S.S. acknowledges NSF DGE 0801620, Integrative Graduate Education and
Research Traineeship (IGERT) on the Integrated Science and Engineering of Stem Cells. We would like to thank Dr. Cheoljin Kim for help with synthesis and Perry Yin for his useful suggestions and comments on the manuscript.

\section{REFERENCES}

(1) Lyssiotis, C. A.; Lairson, L. L.; Boitano, A. E.; Wurdak, H.; Zhu, S.; Schultz, P. G. Angew. Chem., Int. Ed. Engl. 2011, 50, 200.

(2) Schugar, R. C.; Robbins, P. D.; Deasy, B. M. Gene Ther. 2008, 15, $126-35$.

(3) Xu, Y.; Shi, Y.; Ding, S. Nature 2008, 453, 338-344.

(4) Kim, D. H.; Rossi, J. J. Nat. Rev. Genet. 2007, 8, 173-184.

(5) Jung, J. J.; Solanki, A.; Memoli, K. A.; Kamei, K.; Kim, H.; Drahl, M. A.; Williams, L. J.; Tseng, H. R.; Lee, K. Angew. Chem., Int. Ed. 2010, 49, 103-107.

(6) Yau, W. W.; Rujitanaroj, P. O.; Lam, L.; Chew, S. Y. Biomaterials 2012, 33, 2608-28.

(7) Jorgensen, W. L.; Duffy, E. M. Adv. Drug Delivery Rev. 2002, 54, 355-66.

(8) Pal, R.; Mamidi, M. K.; Das, A. K.; Bhonde, R. Arch. Toxicol. 2012, 86, 651-61.

(9) Wang, J.; Lu, Z.; Wientjes, M. G.; Au, J. L. AAPS J 2010, 12, 492-503.

(10) Zhao, F.; Zhao, Y.; Liu, Y.; Chang, X.; Chen, C. Small 2011, 7, $1322-37$.

(11) Cheng, D.; Cao, N.; Chen, J.; Yu, X.; Shuai, X. Biomaterials 2012, 33, 1170-9.

(12) Sun, T.-M.; Du, J.-Z.; Yao, Y.-D.; Mao, C.-Q.; Dou, S.; Huang, S.-Y.; Zhang, P.-Z.; Leong, K. W.; Song, E.-W.; Wang, J. ACS Nano 2011, 5, 1483-1494.

(13) Brewster, M. E.; Loftsson, T. Adv. Drug Delivery Rev. 2007, 59, 645-666.

(14) Davis, M. E.; Brewster, M. E. Nat. Rev. Drug Discovery 2004, 3, 1023-1035.

(15) Yang, S.; May, S. J. Chem. Phys. 2008, 129, 185105.

(16) Pun, S. H.; Bellocq, N. C.; Liu, A. J.; Jensen, G.; Machemer, T.; Quijano, E.; Schluep, T.; Wen, S. F.; Engler, H.; Heidel, J.; Davis, M. E. Bioconjugate Chem. 2004, 15, 831-840.

(17) Kim, C.; Shah, B. P.; Subramaniam, P.; Lee, K.-B. Mol. Pharm. 2011, 8, 1955-1961.

(18) Venkataraman, S.; Hedrick, J. L.; Ong, Z. Y.; Yang, C.; Ee, P. L. R.; Hammond, P. T.; Yang, Y. Y. Adv. Drug Delivery Rev. 2011, 63, $1228-1246$

(19) Davis, M. E.; Bellocq, N. C. J. Incl. Phenom. Macro. 2002, 44, $17-22$.

(20) Solanki, A.; Shah, S.; Yin, P. T.; Lee, K.-B. Sci. Rep. 2013, 3, $1553-1559$.

(21) Stolt, C. C.; Lommes, P.; Sock, E.; Chaboissier, M. C.; Schedl, A.; Wegner, M. Genes Dev. 2003, 17, 1677-89.

(22) Marill, J.; Idres, N.; Capron, C. C.; Nguyen, E.; Chabot, G. G. Curr. Drug Metab. 2003, 4, 1-10.

(23) Leypoldt, F.; Lewerenz, J.; Methner, A. J. Neurochem. 2001, 76, 806-14.

(24) Solanki, A.; Shah, S.; Memoli, K. A.; Park, S. Y.; Hong, S.; Lee, K. B. Small 2010, 6, 2509-13. 